\title{
Prédominance du papillomavirus humain 56 dans une sous-population de femmes sexuellement actives à Garango, Centre-Est, Burkina Faso.
}

\author{
Rogomenoma. Alice Ouedraogo 1, 2, Théodora Mahoukèdè Zohoncon 1,2,3, Silmangdé Patricia \\ Guigma 1,3, Abdoul Karim Ouattara 1,2, et Jacques. Simpore ${ }^{1,2,3}$ \\ 1 Laboratoire de Biologie Moléculaire et de Génétique (LABIOGENE), Université Joseph KI-ZERBO, 03 BP 7021 \\ Ouagadougou 03, Burkina Faso. \\ ${ }^{2}$ Centre de Recherche Biomoléculaire Pietro Annigoni (CERBA), 01 BP 364 Ouagadougou 01, Burkina Faso. \\ 3Faculté de Médicine, Université Saint Thomas d'Aquin, 06 BP 10212 Ouagadougou 01, Burkina Faso. \\ Auteur correspondant : Prof. Jacques Simpore, Centre de Recherche Biomoléculaire Pietro Annigoni (CERBA) / \\ LABIOGENE, Université Joseph KI-ZERBO, 01 BP 364 Ouagadougou 01, Burkina Faso, Afrique de l'Ouest. Tel: \\ +226 25361232, Fax: +226 25363242; jacques.simpore@yahoo.fr; ORCID IDs: 0000-0002-0415-9161.
}

Original submitted in on $8^{\text {th }}$ April 2020. Published online at www.m.elewa.org/journals/ on $30^{\text {th }}$ June 2020 https://doi.org/10.35759/JABs.150.10

\section{RESUME}

Objectif : cette étude a été conduite dans le but d'identifier les génotypes de Papillomavirus Humains à haut risque oncogène (HPV-HR) circulant chez les femmes sexuellement actives à Garango, au Burkina Faso.

Méthodologie et résultats : avant le dépistage des lésions précancéreuses, des échantillons endocervicaux ont été prélevés chez 135 femmes sexuellement actives à Garango. L'ADN extrait a permis de caractériser 14 génotypes de HPV-HR à travers une PCR multiplexe en temps réel. Quarante-trois pourcent (43\%) des femmes portaient une infection à HPV à haut risque oncogène soit 58/135. Sur les quatorze génotypes testés, treize ont été identifiés et le génotype le plus fréquent était le HPV 56 (62,5\%) suivi des HPV 18 (5,5\%), HPV 68 (4,2\%), HPV 66 (4,2\%), HPV 59 (4,2\%), HPV 58 (4,2\%), HPV 35 (4,2\%). Le HPV 33 inclus dans le vaccin anti-HPV n'a pas été identifié chez les femmes de notre étude. Conclusion et application des résultats : Ce type d'étude qui est la première à Garango a montré une forte prévalence du génotype HPV 56 qui n'est pas encore couvert par un vaccin. Ces résultats constituent une contribution scientifique sur l'épidémiologie et la distribution des génotypes HPV-HR et permettront de guider nos politiques de santé vers une meilleure prévention du cancer du col de l'utérus.

Mots-clés : Papillomavirus humain à haut risque, PCR en temps réel, génotypes, femmes, Garango. 


\section{Predominance of Human Papillomavirus 56 in a subpopulation of sexually active women in} Garango, Central-East, Burkina Faso

\section{ABSTRACT}

Objective: The aim of this study was to identify circulating strains of HR-HPV among sexually active women in Garango, Burkina Faso.

Methodology and results: Before screening for precancerous lesions, endocervical samples were taken from 135 sexually active women in Garango. The extracted DNA made it possible to characterize 14 HRHPV genotypes through a real-time multiplex PCR. Forty three percent $(58 / 135)$ of women had a high-risk oncogenic HPV infection. Of the fourteen genotypes tested, thirteen were identified and the most frequent genotype was HPV 56 (62.5\%) followed by HPV 18 (5.5\%), HPV 68 (4.2\%), HPV 66 (4.2\%), HPV 59 (4.2 $\%)$, HPV 58 (4.2\%), HPV35 (4.2\%). The genotype HPV 33 included in the HPV vaccine was not identified in the women in our study.

Conclusion and application of finding: this type of study, which is the first one in Garango, has showed a high prevalence of genotype HPV 56 which is not yet covered by a vaccine. These results constitute a scientific contribution to the epidemiology and distribution of the HR-HPV genotypes and will help guide our health policies towards better prevention of cervical cancer.

Keywords: High-Risk Human Papillomavirus, real time PCR, genotypes, women, Garango

\section{INTRODUCTION}

Les femmes sexuellement actives sont à risque d'infection génitale par le HPV-HR, et la persistance de cette infection est étroitement liée au cancer invasif du col utérin (Johnson et al., 2012), principale cause de décès par cancer chez les femmes en Afrique subsaharienne. A l'échelle mondiale, cette infection est un véritable problème de santé publique. Les vaccins anti-HPV actuellement disponibles sur le marché ne ciblent que quelques génotypes à haut risque oncogène, alors que d'autres génotypes HPV-HR seraient plus fréquents dans la population africaine, en particulier au Burkina Faso et seraient associés au cancer du col utérin. Cependant, au Burkina Faso,

\section{MATERIEL ET METHODES}

Site et population d'étude : D'Avril à Mai 2016, à la suite d'une campagne de sensibilisation sur le dépistage des lésions précancéreuses et du cancer du col de l'utérus, nous avons mené deux études distinctes sur le diagnostic du HPV-HR dans la région sanitaire du Centre-Est sur deux populations différentes de femmes sexuellement actives, en particulier dans les villes de Garango et de Tenkodogo (Ouedraogo et al., 2018). La population cible était des femmes non enceintes sexuellement actives, qui n'avaient pas leurs règles et qui n'avaient pas subi d'hystérectomie totale. En effet, au Centre Médical avec Antenne chirurgicale de Garango (CMA), 135 échantillons cervicaux ont été des études ont rapporté une prédominance d'autres génotypes de HPV-HR (Zohoncon et al., 2013; Traore et al., 2016a, 2016b; Ouedraogo et al., 2018) retrouvés dans des cas de cancer et de lésions précancéreuses (Ouedraogo et al., 2011; Djigma et al., 2011; Zohoncon et al., 2016a, 2016b). Compte tenu de la morbidité et de la mortalité causée par le cancer du col de l'utérus induit par le HPV-HR, l'objectif de cette étude était de contribuer à la cartographie du HPV-HR en identifiant les génotypes circulants et leur prévalence dans une sous-population de femmes sexuellement actives à Garango pour des stratégies de prévention adéquates.

prélevés chez des femmes sexuellement actives et consentantes. Chacune d'elles a répondu à un questionnaire standardisé pour la collecte d'informations sur les caractéristiques sociodémographiques, comportementales et sexuelles, le niveau de connaissances sur le HPV et le cancer du col de l'utérus (Ouedraogo et al., 2011; Djigma et al., 2011; Zohoncon et al., 2013; Ouedraogo et al., 2015; Traore et al., 2016a; Zohoncon et al., 2016a, 2016b). Collecte d'échantillons endocervicaux et dépistage des lésions précancéreuses : À l'aide d'un spéculum à usage unique et d'un écouvillon stérile, des échantillons endocervicaux ont été prélevés chez les 
femmes. Les échantillons collectés ont été congelés dans un milieu de transport à $-20^{\circ} \mathrm{C}$ et envoyés au Centre de Recherche Biomoléculaire Pietro Annigoni (CERBA) de Ouagadougou pour analyse moléculaire. Après l'échantillonnage, le dépistage des lésions précancéreuses a été effectué par inspection visuelle après application d'acide acétique et de Lugol (IVA / IVL).

Considération éthique: Cette étude a reçu l'approbation du Comité d'éthique pour la recherche en santé du Burkina Faso (numéro de délibération 2016-3026) ainsi que celle de la Direction Régionale de la Santé (DRS) de la Région sanitaire du Centre-Est. Toutes les femmes ont donné leur consentement libre et éclairé pour participer à l'étude.

Extraction d'ADN et caractérisation des génotypes du HPV à haut risque oncogène : L'ADN viral a été extrait à l'aide du kit DNA-Sorb-A (Sacace Biotechnologies, Como, Italie) à partir des échantillons

\section{RESULTATS}

Les caractéristiques sociodémographiques des femmes de l'étude : L'âge des femmes variait de 20 à 60 ans avec une moyenne de 39,2 \pm 9,9 ans. La majorité des femmes étaient âgées de plus de 30 ans $(82,2 \%)$, étaient mariées ou vivaient avec un partenaire $(73,3 \%)$. Selon le niveau d'éducation, $17 \%$, $45,2 \%$ et $4,4 \%$ des femmes avaient atteint respectivement l'école primaire, le lycée et l'université. En outre, 33,3\% des femmes étaient analphabètes, $38,5 \%$ étaient des fonctionnaires et $34,1 \%$ étaient des ménagères. L'âge des femmes au moment du premier rapport sexuel variait de 10 à 30 ans avec une moyenne de 19,5 $\pm 2,6$ ans. Les femmes qui avaient plusieurs partenaires sexuels étaient de $12,6 \%$ et 87,4 $\%$ n'avaient qu'un seul partenaire sexuel. Dans cette endocervicaux selon le protocole fourni par le fabricant. La caractérisation des génotypes HPV-HR a été réalisée à l'aide du Sacycler-96 Real Time PCR v.7.3 (SACACE Biotechnologies $囚$, Como, Italie) et du kit "HPV Genotypes 14 Real-TM Quant" (Sacace Biotechnologies, Como, Italie), qui permettait d'identifier quatorze génotypes de HPV-HR $(16,18,31,33,35,39$, $45,51,52,56,58,59,66,68)$ par une PCR multiplex en temps réel. Le programme PCR utilisé était le suivant : 1 cycle de $95^{\circ} \mathrm{C}$ pendant 15 minutes ; 5 cycles de $95^{\circ} \mathrm{C}$ pendant $05 \mathrm{~s}, 60^{\circ} \mathrm{C}$ pendant $20 \mathrm{~s}, 72$ ${ }^{\circ} \mathrm{C}$ pendant $15 \mathrm{~s} ; 40$ cycles de $95^{\circ} \mathrm{C}$ pendant $05 \mathrm{~s}$, $60^{\circ} \mathrm{C}$ pendant $30 \mathrm{~s}$ et $72^{\circ} \mathrm{C}$ pendant $15 \mathrm{~s}$.

L'analyse des données : Les données ont été traitées et analysées à l'aide des logiciels IBM SPSS V.21 (SPSS, Chicago, Illinois, États-Unis) et Epi Info 6. Le test du chi carré a été utilisé pour des comparaisons avec une différence significative pour $p<0,05$.

population de femmes, seulement $6,7 \%$ n'avaient jamais été enceintes. Les autres avaient été enceintes au moins une fois dans leur vie $(93,3 \%)$ et $23,7 \%$ d'entre elles ont eu plus de 5 enfants. Par ailleurs, plus de la moitié des femmes (59,3\%) employaient une méthode contraceptive. De plus, $77 \%$ n'avaient jamais utilisé de préservatif pendant les rapports sexuels, et 83 $\%$ n'avaient aucune connaissance sur le cancer du col de l'utérus et l'infection à HPV au moment de leur inclusion dans l'étude (tableau 1). Le tableau 1 montre également que la répartition des femmes selon les caractéristiques sociodémographiques, sexuelles et comportementales était statistiquement très significative $(P<0,01)$ à l'exception de la profession.

Tableau 1 : Caractéristiques sociodémographiques, sexuelles et comportementales des femmes de l'étude à Garango.

\begin{tabular}{lccc}
\hline Caractéristiques & Effectif & (\%) & $P$-value \\
\hline Tranche d'âge en année & & & $\mathbf{P}<\mathbf{0 , 0 1}$ \\
$<30$ ans & 24 & 17,8 & \\
$\geq 30$ ans & 111 & 82,2 & \\
Niveau d'instruction & & & $\mathbf{P}=\mathbf{0 , 0 2}$ \\
Non instruite & 45 & 33,3 & \\
Primaire & 23 & 17 & \\
Secondaire & 61 & 45,2 & \\
Université & 6 & 4,4 & \\
Situation matrimoniale & & & $\mathbf{P}<\mathbf{0 , 0 1}$ \\
Mariée & 99 & 73,3 & \\
Célibataire & 16 & 11,9 & \\
\hline
\end{tabular}


Ouedraogo et al., J. Appl. Biosci. 2020 Prédominance du papillomavirus humain 56 dans une sous-population de femmes sexuellement actives à Garango, Centre-Est, Burkina Faso.

\begin{tabular}{|c|c|c|c|}
\hline Veuve & 17 & 12,6 & \\
\hline Divorcée & 3 & 2,2 & \\
\hline Nombre de partenaires sexuels & & & $P<0,01$ \\
\hline 1 & 118 & 87,4 & \\
\hline$\geq 2$ & 17 & 12,6 & \\
\hline Profession exercée & & & $P=0,12$ \\
\hline Ménagères & 46 & 34,1 & \\
\hline Elèves/Etudiantes & 11 & 8,1 & \\
\hline Fonctionnaire & 52 & 38,5 & \\
\hline Secteur informel & 26 & 19,3 & \\
\hline Utilisation d'une contraception & & & $P=0,03$ \\
\hline Non/Méthode naturelle & 80 & 59,3 & \\
\hline Oui & 55 & 40,7 & \\
\hline Utilisation du préservatif & & & $P<0,01$ \\
\hline Oui & 31 & 23 & \\
\hline Non & 104 & 77 & \\
\hline Age du premier rapport sexuel & & & $P<0,01$ \\
\hline$<18$ ans & 25 & 18,5 & \\
\hline$\geq 18$ ans & 110 & 81,5 & \\
\hline Parité & & & $P<0,01$ \\
\hline 0 & 9 & 6,7 & \\
\hline $1-5$ & 94 & 69,6 & \\
\hline $5-13$ & 32 & 23,7 & \\
\hline Connaissance du HPV et du cancer du col de l'utérus & & & $P<0,01$ \\
\hline Oui & 23 & 17 & \\
\hline Non & 112 & 83 & \\
\hline
\end{tabular}

Prévalence de l'infection à HPV-HR et caractérisation des génotypes chez les femmes à Garango: Dans cette étude, tous les échantillons testés étaient positifs pour le gène de la $\beta$-globine utilisé comme contrôle interne. Sur les quatorze génotypes HPV-HR recherchés par le kit d'amplification utilisé, treize ont été identifiés. Les résultats de la recherche sur le HPV-HR ont montré que $43 \%$ (58/135) des femmes étaient infectées par au moins un génotype HPV-HR. Le nombre total de génotypes par femme infectée allait de 1 à 4 . La figure 1 montre la distribution de ces génotypes parmi lesquels les plus fréquents étaient en ordre décroissant les HPV $56(62,5$ $\%)$, HPV 18 (5,5\%), HPV 68 (4,2\%), HPV 66 (4,2\%), HPV $59(4,2 \%)$, HPV $58(4,2 \%)$, HPV 35 (4,2\%), HPV $52(2,7 \%)$, HPV $51(2,7 \%)$, HPV $45(1,4 \%)$, HPV 39 $(1,4 \%)$, HPV $31(1,4 \%)$, HPV 16 (1,4\%). Le génotype HPV 33 n'a pas été identifié chez les femmes de cette étude. 


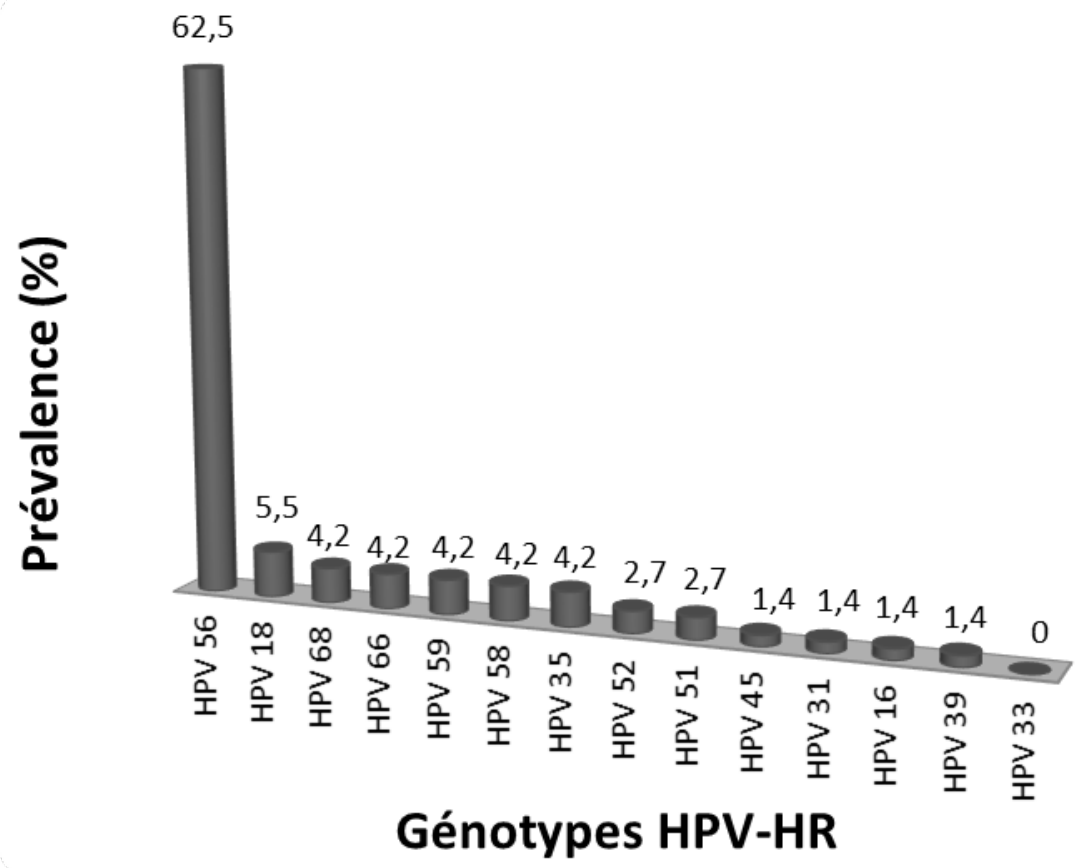

FIGURE 1 : Fréquence des génotypes de HPV-HR identifiés chez les femmes de l'étude

Répartition des génotypes de HPV à haut risque en fonction du type d'infection: Parmi les femmes infectées par le HPV-HR, 84,5\% (49/58) étaient porteuses d'un seul génotype. Les infections multiples étaient de 15,5\% (9/58). Sur les 13 génotypes de HPVHR identifiés chez ces femmes, nous avons dénombré un total de 72 génotypes dont $23(31,9 \%)$ dans les 9 cas d'infections multiples contre $49(68,1 \%)$ dans les infections isolées. II y avait 1,7\% (1/58) de co-infection HPV16 / 18. Le tableau 2 donne la répartition des génotypes HPV-HR en fonction des infections isolées ou multiples.

Tableau 2 : Prévalence et distribution de 14 génotypes HPV-HR dans les infections isolées et multiples chez les femmes à Garango.

\begin{tabular}{lllc}
\hline \multicolumn{2}{l}{ Génotypes HPV-HR associés aux infections isolées (\%) } & $\begin{array}{l}\text { Génotypes HPV-HR associés aux infections } \\
\text { multiples (\%) }\end{array}$ \\
\hline HPV18 & $1(1,7)$ & HPV16+18 & $1(1,7)$ \\
HPV35 & $2(3,5)$ & HPV39+56 & $1(1,7)$ \\
HPV52 & $2(3,5)$ & HPV51+56 & $1(1,7)$ \\
HPV56 & $39(67,2)$ & HPV56+58 & $1(1,7)$ \\
HPV58 & $1(1,7)$ & HPV59+66 & $1(1,7)$ \\
HPV59 & $1(1,7)$ & HPV18+31+56 & $1(1,7)$ \\
HPV66 & $2(3,5)$ & HPV51+56+58 & $1(1,7)$ \\
HPV68 & $1(1,7)$ & HPV56+59+68 & $1(1,7)$ \\
HPV33 & - & HPV18+35+45+68 & $1(1,7)$ \\
Total 1 & $\mathbf{4 9 ( 8 4 , 5 )}$ & Total 2 & $\mathbf{9}(15,5)$ \\
\hline \multicolumn{2}{l}{ Total des génotypes HPV-HR } & $\mathbf{5 8 ( 1 0 0 )}$ \\
\hline
\end{tabular}

Distribution des génotypes de HPV-HR selon la couverture vaccinale : Sur le cumul de 72 génotypes HPV-HR identifiés chez les femmes de notre étude, 6,9 $\%$ étaient couverts par le vaccin bivalent Cervarix® ou Cervarix $^{\top M}$ (GlaxoSmithkline, Rixensart, Belgique/
Medimmune, Maryland) (Cervarix) disponible au Burkina Faso et 16,6 \% étaient couverts par le vaccin nonavalent Gardasil 9 (Merck \& Co, Sanofi Pasteur MSD). Cependant, 83,3\% de ces génotypes n'étaient couverts par aucun vaccin anti-HPV (tableau 3). En 
considérant les génotypes couverts par les vaccins anti-HPV, 3,7 \% (5/135) des femmes avaient le HPV 16 et/ou 18 , soit $8,6 \%(5 / 58)$ de celles infectées par au moins un génotype HPV-HR ; en outre, 8,9\% (12/135) avaient un ou plusieurs des génotypes $16 / 18 / 31 / 33 / 45 / 52 / 58$ soit $20,7 \%(12 / 58)$ de celles infectées.

Tableau 3 : Distribution des génotypes de HPV à haut risque oncogène selon la couverture vaccinale

\begin{tabular}{|c|c|c|c|c|c|}
\hline \multicolumn{2}{|c|}{$\begin{array}{l}\text { Génotypes HPV-HR présents chez } \\
\text { les femmes et couverts par } \\
\text { Cervarix® disponible au Burkina } \\
\text { Faso }\end{array}$} & \multicolumn{2}{|c|}{$\begin{array}{l}\text { Génotypes HPV-HR présents chez } \\
\text { les femmes et couverts par le vaccin } \\
\text { nonavalent }\end{array}$} & \multicolumn{2}{|c|}{$\begin{array}{l}\text { Génotypes HPV-HR présents } \\
\text { chez les femmes et non } \\
\text { couverts par un vaccin anti- } \\
\text { HPV }\end{array}$} \\
\hline HPV-HR & $\mathrm{N}(\%)$ & HPV-HR & $\mathrm{N}(\%)$ & HPV-HR & $\mathrm{N}(\%)$ \\
\hline $\begin{array}{l}16 \\
18\end{array}$ & $\begin{array}{l}1(1,4) \\
4(5,5)\end{array}$ & $\begin{array}{l}16 \\
18 \\
31 \\
45 \\
52 \\
58 \\
33\end{array}$ & $\begin{array}{l}1(1,4) \\
4(5,5) \\
1(1,4) \\
1(1,4) \\
2(2,7) \\
3(4,2) \\
-\end{array}$ & $\begin{array}{l}56 \\
68 \\
66 \\
59 \\
35 \\
51 \\
39\end{array}$ & $\begin{array}{l}45(62,5) \\
3(4,2) \\
3(4,2) \\
3(4,2) \\
3(4,2) \\
2(2,7) \\
1(1,4)\end{array}$ \\
\hline Total & $5(6,9)$ & & $12(16,6)$ & & $60(83,4)$ \\
\hline
\end{tabular}

Facteurs de risque associés au portage du HPVHR: Dans cette étude, nous avons analysé l'association entre l'infection par le HPV-HR et les facteurs de risque tels que l'âge, la parité, l'état matrimonial, les antécédents d'IST, le résultat IVA / IVL, les connaissances sur le cancer du col utérin et le HPV. II n'y avait aucune association statistique entre ces facteurs et l'infection à HPV-HR (tableau 4). Cependant, les premiers rapports sexuels avant l'âge de 18 ans, les partenaires sexuels multiples et l'absence d'utilisation du préservatif lors des rapports sexuels augmenteraient le risque d'infection par le HPV-HR $(P<0,01)$.

Tableau 4 : Facteurs de risque potentiels associés au portage du HPV-HR

\begin{tabular}{|c|c|c|c|c|}
\hline \multirow[t]{2}{*}{ Facteurs de risque } & \multicolumn{3}{|c|}{ Présence du HPV-HR } & \multirow[t]{2}{*}{ P-value } \\
\hline & $\begin{array}{l}\text { HPV - } \\
n=77\end{array}$ & $\begin{array}{l}\text { HPV + } \\
n=58\end{array}$ & $\begin{array}{l}\text { Total } \\
n=135\end{array}$ & \\
\hline $\begin{array}{l}\text { Tranche d'âge, } n(\%) \\
<30 \text { ans } \\
\geq 30 \text { ans }\end{array}$ & $\begin{array}{l}11(45,8) \\
66(59,5)\end{array}$ & $\begin{array}{l}13(54,2) \\
45(40,5)\end{array}$ & $\begin{array}{l}24 \\
111\end{array}$ & 0,221 \\
\hline $\begin{array}{l}\text { Age au moment du premier rapport sexuel, } \boldsymbol{n}(\%) \\
<18 \text { ans } \\
\geq 18 \text { ans }\end{array}$ & $\begin{array}{l}21(84) \\
56(50,9)\end{array}$ & $\begin{array}{l}4(16) \\
54(49,1)\end{array}$ & $\begin{array}{l}25 \\
110\end{array}$ & 0,003 \\
\hline $\begin{array}{l}\text { Nombre de partenaires sexuels, } n(\%) \\
1 \\
\geq 2 \\
\text { Parité, } n(\%) \\
\text { Nullipare } \\
\text { Primipare } \\
\text { Multipare }\end{array}$ & $\begin{array}{l}74(62,7) \\
3(17,6) \\
3(33,3) \\
7(77,8) \\
67(57,3)\end{array}$ & $\begin{array}{l}44(37,3) \\
14(82,4) \\
\\
6(66,7) \\
2(22,2) \\
50(42,7)\end{array}$ & $\begin{array}{l}118 \\
17 \\
9 \\
9 \\
117\end{array}$ & $<0,001$ \\
\hline $\begin{array}{l}\text { Utilisation du préservatif, } n(\%) \\
\text { Oui } \\
\text { Non } \\
\text { Situation matrimoniale, } n(\%)\end{array}$ & $\begin{array}{l}10(32,3) \\
67(64,4)\end{array}$ & $\begin{array}{l}21(67,7) \\
37(35,6)\end{array}$ & $\begin{array}{l}31 \\
104\end{array}$ & 0,001 \\
\hline
\end{tabular}




\begin{tabular}{l|l|l|l|l} 
Célibataire & $6(37,5)$ & $10(62,5)$ & 16 & \\
Mariée & $56(56,6)$ & $43(43,4)$ & 99 & \\
Divorcée & $2(66,7)$ & $1(33,3)$ & 3 & \\
Veuve & $13(76,5)$ & $4(23,5)$ & 17 & \\
Antécédents d'IST, n (\%) & $45(55,6)$ & $36(44,4)$ & 81 & \\
Oui & $32(59,3)$ & $22(40,7)$ & 54 & \\
Non & $77(57)$ & $58(43)$ & 135 & \\
Résultats IVA/IVL, n (\%) & 0 & 0 & 0 & \\
IVA/IVL - & & & & 0,327 \\
IVA/IVL + & $11(47,8)$ & $12(52,2)$ & 23 & \\
Connaissance du HPV/cancer du col de l'utérus, $n(\%)$ & $66(58,9)$ & $46(41,1)$ & 112 & \\
Oui & &
\end{tabular}

${ }^{*} \mathrm{NA}$ : non applicable

\section{DISCUSSION}

La taille de notre échantillon ne nous permet pas de généraliser. Malgré les limites de cette étude, elle était la première à Garango, ville située dans une zone à risque d'IST du fait de la prévalence élevée de l'infection à VIH $(0,9 \%$; INSD, 2010) et notamment du fait que cette région frontalière abrite des marchés à renommée internationale favorisant un important brassage des populations et l'expansion des IST, du $\mathrm{VIH} / \mathrm{SIDA}$ et les flambées épidémiques. Elle nous apporte non seulement des résultats complémentaires pour évaluer le niveau d'infection à HPV-HR et de donner des informations sur sa répartition au Burkina Faso.

Prévalence des infections HPV-HR: La prévalence de l'infection à HPV-HR dans cette population d'étude était comparable à celle trouvée chez les adolescentes à Ouagadougou au Burkina Faso (Ouedraogo et al., 2015). Cependant, elle était plus élevée que celles trouvées au Ghana (Obiri-Yeboah et al., 2017) ; au Burkina Faso (Traore et al., 2016a, 2016b; Ouedraogo et al., 2018) ; au Costa Rica (Chaturvedi et al., 2011); au Bénin (Piras et al., 2011) ; en Grande-Bretagne (Johnson et al., 2012) et en Italie (Campari et al., 2015). Bien que la prévalence de l'infection génitale à HPV varie d'une région à l'autre dans le monde (Clifford et al., 2006), toutes les études conviennent qu'elle est particulièrement importante chez les jeunes femmes au début de l'activité sexuelle et diminue plus tard lorsque l'âge augmente, en raison à la fois de la clairance virale et de l'acquisition d'immunité. La forte prévalence de l'infection à HPV-HR dans cette étude pourrait s'expliquer par le fait que dix-sept femmes avaient plusieurs partenaires sexuels et plus de quatre-vingt pour cent d'entre elles étaient infectées par le HPV-HR.
Caractérisation du HPV-HR : Dans la présente étude, le génotype le plus fréquent chez les femmes était le HPV 56. Des études similaires sur le HPV menées au Burkina Faso et au Vietnam ont montré des génotypes différents et prédominants autres que le HPV 56 (Ouedraogo et al., 2015; Traore et al., 2016a, 2016b; Zohoncon et al., 2016a,2016b; Van et al., 2017). Cependant, le HPV 56 semble être le plus répandu dans la région Centre-Est du Burkina Faso (Ouedraogo et al., 2018). Dans quatre-vingt pour cent des cas, on observe une clairance virale du HPV mais la persistance de l'infection peut conduire à une néoplasie intraépithéliale cervicale (CIN) qui peut régresser ou évoluer vers un cancer du col de l'utérus (CCU). En outre, selon la littérature, contrairement aux résultats de cette étude, le HPV 56 n'est généralement pas le plus prédominant mais a déjà été identifié à Ouagadougou et au Ghana chez des femmes séropositives (Zohoncon et al., 2013; Obiri-Yeboah et al., 2017), des adolescentes (Ouedraogo et al., 2015) et surtout dans des cas de CIN2 ou CIN3 (Zohoncon et al., 2016b). De plus, les génotypes HPV-HR retrouvés chez les femmes à Garango (HPV $56 ; 18 ; 59 ; 58 ; 35 ; 52 ; 51$ ; 45 ; 39 ; 31) avaient déjà été identifiés comme les plus fréquents dans des cas de $\mathrm{CIN}$ et $\mathrm{CCU}$ à Ouagadougou, à Parakou et au Ghana (Zohoncon et al., 2016a, 2016b; Obiri-Yeboah et al., 2017) alors que certains de ces génotypes fréquents ne sont pas couverts par les vaccins disponibles. Par ailleurs, plus de la moitié des génotypes HPV-HR trouvés chez les femmes de notre étude ainsi que le génotype 56 le plus fréquent ne sont inclus dans aucun vaccin anti-HPV. A partir de ce constat, faut-il comprendre que les femmes de la ville de Garango, quel que soit leur âge, même si elles ont été vaccinées à l'adolescence par Cervarix TM 
ou Gardasil@, ne seront pas protégées contre les génotypes les plus répandu dans leur population?

Parmi les femmes recrutées à Garango, la prévalence des HPV 16 et 18 couverts par les vaccins actuellement disponibles était très faible. La prévalence du HPV18 obtenue était similaire à celle de l'étude de Johnson et al., 2012 (Johnson et al., 2012) mais inférieure à celles trouvées en Afrique du Sud (Dols et al., 2012); à Ouagadougou (Ouedraogo et al., 2011) et à BoboDioulasso (Traore et al., 2016a). Nos résultats diffèrent de ceux de la littérature selon laquelle les génotypes du HPV-16/18 sont les plus fréquents dans la plupart des régions du monde notamment dans les cas de cancer (Clifford et al., 2006; Bruni et al., 2010; Dols et al., 2012; Heard et al., 2013; Panatto et al., 2013; Tornesello et al., 2014; Guettiti et al., 2014; Monsonego et al., 2015) .Par ailleurs, nos résultats corroborent ceux d'autres études qui avaient déjà montré une régression de la prévalence du HPV 16 dans certains pays africains au profit d'autres génotypes HPV-HR Sagna et al., 2010 ; Bruni et al., 2010 ; Zohoncon et al., 2013; Ouedraogo et al., 2015). En effet, au Burkina Faso, l'étude de Zohoncon et al., (2016b) sur le cancer invasif du col de l'utérus avait révélé une prédominance des HPV 18, 31, 39, 16, 45, 35 et 58. De plus, deux études similaires menées au Bénin et au Burkina Faso dans des cas de néoplasie intraépithéliale cervicale de haut grade et de cancer du col de l'utérus ont montré une absence du HPV 16 et une prédominance de génotypes oncogènes, $18,45,35,52,33,51,31,58$, $66,68,56,59,68$. Bien que les HPV 16 et 18 semblent être les plus répandus dans le monde et en particulier dans les cas de cancer du col de l'utérus, ces études dans la sous-région africaine ont montré une différence régionale dans la distribution des HPV-HR et l'existence de génotypes à haut risque oncogènes prédominants (HPV 56, 51, 59, 68, 66, 39,35) non couvert par les vaccins disponibles. Cette différence classique dans la distribution des génotypes dans notre population d'étude devrait susciter une étude plus large avec un échantillon plus représentatif, afin de déterminer si la fréquence des HPV 16 et 18 chez les femmes est inférieure à celle des autres populations et de caractériser les génotypes oncogènes prédominants dans la sous-région et en particulier dans le cancer du col de l'utérus. Quant aux génotypes de HPV-HR, nos résultats corroborent ceux de (Ouedraogo et al., 2011; Zohoncon et al., 2013) qui ont trouvé que les génotypes HPV de la famille 50'S étaient les plus fréquents chez les femmes à Ouagadougou. Ces résultats semblent confirmer l'importance croissante des génotypes de la famille 50 'S dans les différentes régions du monde et notamment au Burkina Faso. Aussi, la prévalence des infections multiples dans notre population de femmes infectées à Garango était proche de celle rapportée par Ouedraogo et al en 2011 à Ouagadougou mais elle était inférieure à celles trouvées dans les études de Monsonego et al., en 2015, Pannatto et al., (2013) (Panatto et al., 2013) et Kavanagh et al., (2013) (Kavanagh et al., 2013). Dans les infections multiples, le nombre de génotypes (deux à quatre) par femme infectée était comparable à ceux de Traoré et al., (2016a) et Ouedraogo et al., (2015), qui ont trouvé respectivement deux à trois et deux à cinq génotypes HPV-HR dans les cas d'infections multiples. Mais nous avons trouvé une coïnfection HPV 16 / 18, contrairement aux études de Traoré et al., (2016), Ouedraogo et al., (2015) et Pannatto et al., (2013).

Facteurs associés à l'infection par le HPV-HR : II est clair dans la littérature que plusieurs facteurs pourraient contribuer à la persistance de l'infection à HPV. Mais dans cette étude, l'infection à HPV-HR n'était pas associée aux facteurs de risque tels que l'âge, la parité, l'état matrimonial et les antécédents d'IST. Nos résultats sont similaires à ceux de Traoré et al., (2016a) et Ouedraogo et al.,, (2015) mais différent de celui de Pannatto et al.,, (2013) qui ont trouvé une association significative entre l'âge et le résultat HPV positif ou négatif des femmes de leurs études. Cette différence pourrait s'expliquer par le fait que leurs études incluaient à la fois des génotypes de HPV à haut risque et à bas risque oncogène, alors que dans notre étude, nous n'avons considéré que les génotypes à haut risque oncogène. Par ailleurs, certains auteurs ont montré que la prévalence est inversement proportionnelle à l'âge de la patiente (Agorastos et al., 2004). En effet, cette répartition a été retrouvée dans cette étude avec une prévalence de plus de cinquante pour cent avant l'âge de trente ans. Aussi, le nombre de partenaires sexuels des femmes était statistiquement associé à la prévalence du HPV dans cette étude. Ce résultat corrobore celui de la littérature selon lequel le nombre de partenaires sexuels est l'un des principaux facteurs de risque d'infection à HPV (Didelot-Rousseau et al., 2006 ; Ramanakumar et al., 2010). L'absence d'utilisation du préservatif lors les rapports sexuels et la prévalence du HPV-HR étaient statistiquement associés. De plus, l'infection à HPV-HR était associée à l'âge au moment du premier rapport sexuel avant dix-huit ans, ce qui constitue une période de vulnérabilité chez la jeune femme. Les résultats de cette étude n'ont certes pas pu être généralisés à 
l'ensemble de la population de Garango mais ils permettront de connaître les génotypes présents dans une population de femmes de cette ville. Nos résultats montrent la nécessité d'une cartographie HPV-HR au Burkina Faso dans des populations plus

\section{CONCLUSION}

Ce type d'étude, qui est une première à Garango, a montré une forte prévalence du génotype HPV 56 qui n'est pas encore couvert par un vaccin. Cependant, la protection des femmes pourrait s'étendre avec l'introduction du vaccin nonavalent au Burkina Faso. Ces résultats constituent une contribution scientifique sur l'épidémiologie et la distribution des génotypes

\section{REMERCIEMENTS}

Ce travail a été soutenu par la Conférence Episcopale Italienne (CEI), le CERBA / LABIOGENE de l'Université de Ouagadougou et l'Hôpital Saint Camille de

\section{REFERENCES BIBLIOGRAPHIQUES}

Agorastos, T., K. Dinas, B. Lloveras, F. X. Bosch, J. R. Kornegay, J. N. Bontis, and S. de Sanjose. 2004. "Cervical human papillomavirus infection in women attending gynaecological outpatient clinics in northern Greece." Eur J Cancer Prev no. 13 (2):145-7.

Bruni, L., M. Diaz, X. Castellsague, E. Ferrer, F. X. Bosch, and S. de Sanjose. 2010. "Cervical human papillomavirus prevalence in 5 continents: meta-analysis of 1 million women with normal cytological findings." J Infect Dis no. 202 (12): 1789-99.

Campari, C., C. Fedato, A. Petrelli, M. Zorzi, C. Cogo, A. Caprioglio, F. Gallo, L. Giordano, S. Domenighini, L. Pasquale, S. Prandi, M. Zappa, P. Giorgi Rossi, and G. ISCi Migrant Working Group. 2015. "HPV prevalence and risk of pre-cancer and cancer in regular immigrants in Italy: results from HPV DNA testbased screening pilot programs." Infect Agent Cancer no. 10:14. doi: 10.1186/s13027-0150009-x.

Chaturvedi, A. K., H. A. Katki, A. Hildesheim, A. C. Rodriguez, W. Quint, M. Schiffman, L. J. Van Doorn, C. Porras, S. Wacholder, P. Gonzalez, M. E. Sherman, and R. Herrero. 2011. "Human papillomavirus infection with multiple types: pattern of coinfection and risk of cervical disease." J Infect Dis no. 203 (7) :910-20. représentatives, notamment dans des cas de cancer, pour une meilleure politique de prévention du cancer invasif du col utérin.

HPV-HR et permettront de guider nos politiques de santé vers une meilleure prévention du cancer du col de l'utérus. Cette étude montre que la cartographie HPV-HR dans des populations plus représentatives, en particulier dans les cas de cancer, serait nécessaire dans toutes les régions du pays et en Afrique de I'Ouest.

Ouagadougou pour la collecte, l'analyse, l'interprétation des données et la rédaction de cet article.

Clifford, G. M., M. A. Goncalves, S. Franceschi, HPV, and H. I. V. Study Group. 2006. "Human papillomavirus types among women infected with HIV: a meta-analysis." AIDS no. 20 (18):2337-44. doi : 10.1097/01.aids.0000 253361.63578.14.

Didelot-Rousseau, M. N., N. Nagot, V. CostesMartineau, X. Valles, A. Ouedraogo, I. Konate, H. A. Weiss, P. Van de Perre, P. Mayaud, M. Segondy, and Group Yerelon Study. 2006. "Human papillomavirus genotype distribution and cervical squamous intraepithelial lesions among high-risk women with and without HIV1 infection in Burkina Faso." $\mathrm{Br} J$ Cancer no. 95 (3) :355-62. Doi : 10.1038/sj.bjc.6603252.

Djigma, F. W., C. Ouedraogo, D. S. Karou, T. Sagna, C. Bisseye, M. Zeba, D. Ouermi, C. Gnoula, V. Pietra, N. W. Ghilat-Avoid-Belem, K. Sanogo, J. Sempore, S. Pignatelli, A. M. Ferri, J. B. Nikiema, and J. Simpore. 2011. "Prevalence and genotype characterization of human papillomaviruses among HIV-seropositive in Ouagadougou, Burkina Faso." Acta Trop no. 117 (3):202-6. doi : 10.1016/j.actatropica.2010.12.007.

Dols, J. A., G. Reid, J. M. Brown, H. Tempelman, T. R. Bontekoe, W. G. Quint, and M. E. Boon. 2012. "HPV Type Distribution and Cervical Cytology among HIV-Positive Tanzanian and South 
African Women." ISRN Obstet Gynecol no. 2012:514146. doi: 10.5402/2012/514146.

Guettiti, H., E. Ennaifer, L. Attia, D. Chelly, N. B. Alaya, R. B. Aissa, T. Laassili, and S. Boubaker. 2014. "Pre-vaccination prevalence and genotype distribution of human papillomavirus infection among women from urban Tunis: a cross-sectional study." Asian Pac J Cancer Prev no. 15 (21): :9361-5.

Heard, I., L. Tondeur, L. Arowas, M. Falguieres, M. C. Demazoin, and M. Favre. 2013. "Human papillomavirus types distribution in organised cervical cancer screening in France." PLoS One no. 8 (11):e79372. doi: 10.1371/journal.pone.0079372.

Johnson, A. M., C. H. Mercer, S. Beddows, N. de Silva, S. Desai, R. Howell-Jones, C. Carder, P. Sonnenberg, K. A. Fenton, C. Lowndes, and K. Soldan. 2012. "Epidemiology of, and behavioural risk factors for, sexually transmitted human papillomavirus infection in men and women in Britain." Sex Transm Infect no. 88 (3):212-7. doi: 10.1136/sextrans-2011050306 .

Kavanagh, K., K. Sinka, K. Cuschieri, J. Love, A. Potts, K. G. Pollock, H. Cubie, M. Donaghy, and C. Robertson. 2013. "Estimation of HPV prevalence in young women in Scotland; monitoring of future vaccine impact." BMC Infect Dis no. 13:519. doi: 10.1186/1471-233413-519.

Monsonego, J., J. T. Cox, C. Behrens, M. Sandri, E. L. Franco, P. S. Yap, and W. Huh. 2015. "Prevalence of high-risk human papilloma virus genotypes and associated risk of cervical precancerous lesions in a large U.S. screening population: data from the ATHENA trial." Gynecol Oncol no. 137 (1) :47-54.

Obiri-Yeboah, D., P. K. Akakpo, M. Mutocheluh, E. Adjei-Danso, G. Allornuvor, D. Amoako-Sakyi, Y. Adu-Sarkodie, and P. Mayaud. 2017a. "Epidemiology of cervical human papillomavirus (HPV) infection and squamous intraepithelial lesions (SIL) among a cohort of HIV-infected and uninfected Ghanaian women." BMC Cancer no. 17 (1):688. doi: 10.1186/s12885-017-3682-x.

Ouedraogo, C. M., F. W. Djigma, C. Bisseye, T. Sagna, M. Zeba, D. Ouermi, S. D. Karou, V. Pietra, F. Buelli, N. W. Ghilat-Avoid-Belem, K. Sanogo, J. Sempore, R. Moret, S. Pignatelli, J. B.
Nikiema, and J. Simpore. 2011. "[Epidemiology, characterization of genotypes of human papillomavirus in a population of women in Ouagadougou]." J Gynecol Obstet Biol Reprod (Paris) no. 40 (7):633-8. doi: 10.1016/j.jgyn.2011.05.012.

Ouedraogo, C. M., R. M. Rahimy, T. M. Zohoncon, F. W. Djigma, A. T. Yonli, D. Ouermi, A. Sanni, J. Lankoande, and J. Simpore. 2015. "[Epidemiology and characterization of highrisk genotypes of human Papillomavirus in a population of sexually active adolescents in Ouagadougou]." J Gynecol Obstet Biol Reprod (Paris) no. 44 (8) :715-22. doi: 10.1016/j.jgyn.2014.12.021.

Ouedraogo, R. A., T. M. Zohoncon, S. P. Guigma, I. M. Angele Traore, A. K. Ouattara, M. Ouedraogo, F. W. Djigma, D. Obiri-Yeboah, C. Ouedraogo, and J. Simpore. 2018. "Oncogenic human papillomavirus infection and genotypes characterization among sexually active women in Tenkodogo at Burkina Faso, West Africa." Papillomavirus Res no. $6: 22-26$. doi: 10.1016/j.pvr.2018.09.001.

Panatto, D., D. Amicizia, E. Tanzi, S. Bianchi, E. R. Frati, C. M. Zotti, P. L. Lai, A. Bechini, S. Rossi, and R. Gasparini. 2013. "Prevalence of human papillomavirus in young Italian women with normal cytology: how should we adapt the national vaccination policy?" BMC Infect Dis no. 13:575.

Piras, F., M. Piga, A. De Montis, A. R. Zannou, L. Minerba, M. T. Perra, D. Murtas, M. Atzori, M. Pittau, C. Maxia, and P. Sirigu. 2011. "Prevalence of human papillomavirus infection in women in Benin, West Africa." Virol J no. 8:514.

Ramanakumar, A. V., O. Goncalves, H. Richardson, P. Tellier, A. Ferenczy, F. Coutlee, and E. L. Franco. 2010. "Human papillomavirus (HPV) types 16, 18, 31, 45 DNA loads and HPV-16 integration in persistent and transient infections in young women." BMC Infect Dis no. 10:326.

Sagna, T., F. Djigma, M. Zeba, C. Bisseye, S. D. Karou, D. Ouermi, V. Pietra, C. Gnoula, K. Sanogo, J. B. Nikiema, and J. Simpore. 2010. "Human papillomaviruses prevalence and genital coinfections in HIV-seropositive women in Ouagadougou (Burkina Faso)." Pak J Biol Sci no. 13 (19):951-5. 
Tornesello, M. L., P. Giorgi Rossi, L. Buonaguro, F. M. Buonaguro, and H. P. V. Prevalence Italian Working Group. 2014. "Human Papillomavirus Infection and Cervical Neoplasia among Migrant Women Living in Italy." Front Oncol no. 4 :31. doi: $10.3389 /$ fonc. 2014.00031.

Traore, I. M. A., T. M. Zohoncon, O. Ndo, F. W. Djigma, D. Obiri-Yeboah, T. R. Compaore, S. P. Guigma, A. T. Yonli, G. Traore, P. Ouedraogo, C. M. R. Ouedraogo, Y. Traore, and J. Simpore. 2016b. "Oncogenic Human Papillomavirus Infection and Genotype Characterization among Women in Orodara, Western Burkina Faso." Pak J Biol Sci no. 19 (7):306-311. doi: 10.3923/pjbs.2016.306.311.

Traore, I. M., T. M. Zohoncon, A. Dembele, F. W. Djigma, D. Obiri-Yeboah, G. Traore, M. Bambara, C. Ouedraogo, Y. Traore, and J. Simpore. 2016a. "Molecular Characterization of High-Risk Human Papillomavirus in Women in Bobo-Dioulasso, Burkina Faso." Biomed Res Int no. 2016:7092583. doi: 10.1155/2016/7092583.

Van, S. N., M. N. Khac, J. Dimberg, A. Matussek, and A. J. Henningsson. 2017. "Prevalence of Cervical Infection and Genotype Distribution of Human Papilloma Virus among Females in $\mathrm{Da}$ Nang, Vietnam." Anticancer Res no. 37 (3):1243-1247. doi:
Zohoncon, T. M., C. Bisseye, F. W. Djigma, A. T. Yonli, T. R. Compaore, T. Sagna, D. Ouermi, C. M. Ouedraogo, V. Pietra, J. B. Nikiema, S. A. Akpona, and J. Simpore. 2013. "Prevalence of HPV High-Risk Genotypes in Three Cohorts of Women in Ouagadougou (Burkina Faso)." Mediterr J Hematol Infect Dis no. 5 (1) :e2013059.

Zohoncon, T. M., T. C. Ouedraogo, L. V. C. Brun, D. Obiri-Yeboah, W. F. Djigma, S. Kabibou, S. Ouattara, M. Gomina, A. T. Yonli, Vjte Bazie, C. Ouedraogo, O. Lompo, S. A. Akpona, and J. Simpore. 2016a. "Molecular Epidemiology of High-Risk Human Papillomavirus in HighGrade Cervical Intraepithelial Neoplasia and in Cervical Cancer in Parakou, Republic of Benin." Pak J Biol Sci no. 19 (2) :49-56. doi: 10.3923/pjbs.2016.49.56.

Zohoncon, T. M., Prosper Bado, Djénéba Ouermi, Esther M. A. Traoré, Florencia W. Djigma, Ina M. A. Traore, Albert T.Yonli, Charlemagne Ouédraogo, Simon A. Akpona, Olga Lompo, and Jacques Simpore. 2016b. "Human papillomavirus genotypes involved in invasive cervical cancer from formalin-fixed, paraffin embedded tissues in Ouagadougou, Burkina Faso." International Journal of Current Research no. 8 (9) :39314-39318. 